\title{
Factoring and Solving Linear Partial Differential Equations
}

\author{
D. Grigoriev, Rennes, and F. Schwarz, Sankt Augustin
}

\begin{abstract}
The problem of factoring a linear partial differential operator is studied. An algorithm is designed which allows one to factor an operator when its symbol is separable, and if in addition the operator has enough right factors then it is completely reducible. Since finding the space of solutions of a completely reducible operator reduces to the same for its right factors, we apply this approach and execute a complete analysis of factoring and solving a second-order operator in two independent variables. Some results on factoring third-order operators are exhibited.
\end{abstract}

AMS Subjext Classifications: 35A25, 35C05, 35G05.

Keywords: partial differential equations, factorization.

\section{Introduction}

An algorithm for factoring a linear ordinary differential operator $L \in \mathbf{Q}(x)\left[\partial_{x}\right]$ is described in [15], it was improved in [5] with a better complexity bound. In [17] an implementation in a computer algebra system is given. A survey on the factorization problem and further references may be found in [14]. The structure of all possible factorizations of an ordinary differential operator is known due to a fundamental theorem of Loewy [12]. An ordinary operator has a unique factorization into completely reducible factors. Factorization of an operator $L$ is of practical importance because it reduces the problem of finding solutions of the linear differential equation $L v=0$ to the same problem for its factors.

Much less is known on factoring linear partial differential operators (LPDO). First of all, the concept of a completely reducible operator has to be generalized suitably. In the articles by Blumberg [1] and Miller [13] these problems are discussed, and are illustrated by a few typical examples. In particular, an example of a third-order operator is given in [1] which has two different factorizations into completely reducible factors, see Example 4 in Section 4 for a complete discussion. It shows that the result of Loewy quoted above does not remain true for partial differential operators.

In recent times, due to the growing interest in Computer Algebra, a few papers have appeared which treat factoring as finding superideals of a left ideal in the ring of LPDO rather than factoring a single LPDO, see $[20,11]$. In [20] a concept of a factorization is developed which makes some characteristics of factors to be uniquely defined similar to the case of ordinary operators. In [11] the factorization of systems of LPDO with a finite-dimensional (over the subfield of constants) space of solutions is studied, then its linear differential subvarieties are viewed as the factors of the input system.

As in the case of polynomial factoring, an important issue in factoring differential operators is the choice of a ground differential field $F$. If one takes $F$ to be universal [10] then in the case of ordinary operators $L \in F[\partial]$ of order $r$ where $\partial$ is a derivative operator, $L$ has always a factorization in $r$ firstorder factors. This reminds of factoring a univariate polynomial in linear factors over an algebraically closed field. In the case of a LPDO $L \in \mathcal{D}=F\left[\partial_{1}, \ldots, \partial_{m}\right]$ where $\partial_{i}=\partial / \partial x_{i}$ for $i=1, \ldots, m$ denote the derivative operators, one can talk about absolute factorization which resembles the multivariable 
polynomial factorization over an algebraically closed field. On the other hand, the conditions for reducibility of $L$ are rather involved, in general they consist of non-linear partial differential equations. Consequently a large part of the discussion will assume coefficients from a universal field $F$ of zero characteristic. Another advantage of this assumption is the duality between differential varieties and left ideals in $\mathcal{D}$ [10]. On the other hand, studying factorization algorithms we will assume that the input operators are from the ring $\mathbf{Q}\left(x_{1}, \ldots, x_{m}\right)\left[\partial_{1}, \ldots, \partial_{m}\right]$. The simplest possible equations determining the factors are obtained, and its solutions either in $\mathbf{Q}\left(x_{1}, \ldots, x_{m}\right)$ or some extension field are discussed.

Now we say few words about the further contents of the paper whose main purpose is to make some steps towards algorithms for factoring a LPDO and solving the corresponding differential equation.

In Section 1 we study separable LPDO, i. e. one with a separable symbol, and show that one can factor a separable LPDO by means of a procedure similar to Hensel lifting (we name it Hensel descent). Also we define a completely reducible LPDO and establish that if a separable LPDO has enough irreducible right factors then it is completely reducible. The significance of completely reducible LPDO is justified by the result of $[2,18]$ (see the Remark 1.8 below) that the space of solutions of a completely reducible LPDO coincides with the sum of the spaces of its irreducible right factors.

This general approach is applied in Sections 2, 3, 4 to accomplish a complete analysis of factoring a second- (see Section 3) and third-order LPDO (see Section 4) from $F\left[\partial_{x}, \partial_{y}\right]$ respectively, and investigate the solutions of the corresponding differential equations. In Section 2 we consider the intersection of principal ideals generated by first-order LPDO. In the Appendix we give a description of the solutions of partial linear and of partial Riccati differential equations. Evidently, since calculations (even with the aid of a computer) become quite cumbersome, a further theoretical breakthrough in the LPDO factoring is badly needed.

\section{Hensel Descent and Completely Reducible Differential Operators}

For a derivative $\partial^{J}=\partial_{1}^{j_{1}} \cdots \partial_{m}^{j_{m}}$ denote its order $\operatorname{ord}\left(\partial^{J}\right)=|J|=j_{1}+\ldots+j_{m}$. For a LPDO

$$
L=\sum_{|J|=r} a_{J} \partial^{J}+\sum_{|J|<r} b_{J} \partial^{J}
$$

of the order $\operatorname{ord}(L)=r$ the homogeneous polynomial $s(L)=\sum a_{J} Z^{J} \in F\left[Z_{1}, \ldots, Z_{m}\right]$ of degree $r$, with $Z_{k}$ new algebraic indeterminates, is named the symbol of $L$.

Definition 1.1 We call $L$ separable if $s(L)$ is separable.

We observe that if $L$ is separable then in order to factor $L$ one can apply a procedure which we name Hensel descent and which reduces to polynomial factoring (over $F$ ), rational operations in $F$ and taking derivatives. This algorithm is close in nature to the well-known Hensel lifting used widely in polynomial factoring, see e. g. [8], the main difference is that in the case of differential operators one has to compute the coefficients starting with the highest derivatives going to the lowest because in the product of operators the coefficients at higher derivatives of factors give a contribution to the coefficients at lower derivatives of the product.

Thus, let $s(L)=g h$ where $g=\sum_{J} g_{J} Z^{J}, h=\sum_{J} h_{J} Z^{J} \in F\left[Z_{1}, \ldots, Z_{m}\right]$ are homogeneous polynomials of the degrees $\operatorname{deg}(g)=k, \operatorname{deg}(h)=l, k+l=r$. Hensel descent is looking for a factorization in the form

$$
L=\left(\sum_{|J|=k} g_{J} \partial^{J}+\sum_{0 \leq j \leq k-1} G_{j}\right)\left(\sum_{|J|=l} h_{J} \partial^{J}+\sum_{0 \leq j \leq l-1} H_{j}\right)
$$


where $G_{j}=\sum_{|J|=j} g_{J, j} \partial^{J}, H_{j}=\sum_{|J|=j} h_{J, j} \partial^{J}$ contain only the derivatives of order $j$. Denote the corresponding homogeneous polynomials of degrees $j$ by $g_{j}=\sum_{|J|=j} g_{J, j} Z^{J}, h_{j}=\sum_{|J|=j} h_{J, j} Z^{J}$.

Hensel descent runs recursively decreasing the order. Suppose that $G_{j_{1}}, H_{j_{2}}$ are already constructed with $j_{1} \geq \max \{t-l+1,0\}, j_{2} \geq \max \{t-k+1,0\}$ for a certain $0 \leq t \leq r-1$ (at the first step of the recursion we set $t=r-1$ ). At the current step Hensel descent compares the coefficients at the derivatives of order $t$ in both sides of (1). Rewriting the right-hand side in terms of the corresponding homogeneous polynomials of degree $t$ we obtain $g h_{t-k}+h g_{t-l}+p$ (provided that $t \geq k, t \geq l$ ) where the coefficients of the homogeneous polynomial $p$ are already known being the rational expressions of the derivatives of the coefficients of the already constructed $G_{j_{1}}, H_{j_{2}}$.

Taking into account that $t-k<l$ and that due to the separability of $L$ the polynomials $g, h$ are relatively prime, we conclude that there exists at most one pair of polynomials $g_{t-l}, h_{t-k}$ which yields a known polynomial $q=g h_{t-k}+h g_{t-l}$. Hensel descent looks for $g_{t-l}, h_{t-k}$ by means of solving a linear algebraic system in the coefficients of $g_{t-l}, h_{t-k}$. If this system is unfeasible then Hensel descent halts saying that the polynomial factorization $s(L)=g h$ does not lead to a factorization of $L$. Otherwise, Hensel descent outputs $g_{t-l}, h_{t-k}$ and continues the recursion.

In case when $t<k$ (or $t<l$, respectively) the polynomial $h_{t-k}$ is absent (or $g_{t-l}$ is absent, respectively). Finally, in case when both $t<k, t<l$ Hensel descent verifies whether the coefficients at the derivatives of the order $t$ in both sides of (1) coincide. And again it halts if this fails.

Let us summarize the properties of Hensel descent in the following proposition.

Proposition 1.2 A separable LPDO L of order $r$ has at most $2^{r}$ factorizations and Hensel descent finds all of them starting with factoring the polynomial $s(L)$ and performing rational operations and derivations in F. For each (including reducible) factor of $s(L)$ there is at most one right factor of $L$.

Remark 1.3 If $s(L)=g h$ where $g, h$ being relatively prime, one can still apply Hensel descent to (not necessary separable) $L$.

For a LPDO $L$ we denote by $\langle L\rangle$ the left ideal generated by $L$. Since we consider only left ideals, the term left in general will be omitted.

In the ring $\mathcal{D}=F[\partial]$ of ordinary differential operators all ideals are principal. As a consequence the lcm of any two operators $L_{1}, L_{2} \in \mathcal{D}$ is defined uniquely as the operator $L$ generating the intersection $<L_{1}>\cap<L_{2}>$. For LPDO the intersection ideal of two principal ideals is not necessarily principal, that is why we introduce the following concept.

Definition 1.4 A LPDO $L$ is called completely reducible if $\langle L\rangle=<L_{1}>\cap \cdots \cap<L_{k}>$ for suitable irreducible $L P D O L_{1}, \ldots, L_{k}$. In this case we say that $L$ is the least common left multiple $L=l \mathrm{~cm}\left\{L_{1}, \ldots, L_{k}\right\}$. More generally, we call a left ideal $I \subset \mathcal{D}$ completely reducible if $I=<L_{1}>$ $\cap \cdots \cap<L_{k}>$.

Theorem 1.5 If a LPDO $L$ has right factors $L_{1}, \ldots, L_{k}$ such that $l c m\left\{s\left(L_{1}\right), \ldots, s\left(L_{k}\right)\right\}=s(L)$ then $<L>=<L_{1}>\cap \cdots \cap<L_{k}>$.

Proof. Clearly $<L>\subset<L_{1}>\cap \cdots \cap<L_{k}>$. We need to prove the inverse inclusion.

Making an appropriate linear (over the subfield of constants of $F$ ) transformation of $\partial_{1}, \ldots, \partial_{m}$, one can assume that the derivative $\partial_{1}^{r}$ occurs in $L$ (cf. Lemma 2.3 [18] or Lemma 4 [6]).

Take any $P \in<L_{1}>\cap \cdots \cap<L_{k}>$ and divide it from the left with the remainder by $L$ with respect to $\partial_{1}$, then $P=Q L+R$ where $\operatorname{ord}_{\partial_{1}}(R)<r$. Since $R \in<L_{1}>\cap \cdots \cap<L_{k}>$ we have $s(L)=l c m\left\{s\left(L_{1}\right), \ldots, s\left(L_{k}\right)\right\} \mid s(R)$ and we get a contradiction because $r=\operatorname{deg}_{Z_{1}}(s(L))>\operatorname{deg}_{Z_{1}}(s(R))$ unless $R=0$. 
Corollary 1.6 1) If $L$ has irreducible right factors $L_{1}, \ldots, L_{k}$ such that $l c m\left\{s\left(L_{1}\right), \ldots, s\left(L_{k}\right)\right\}=$ $s(L)$ then $\left\langle L>=<L_{1}>\cap \cdots \cap<L_{k}>\right.$ is completely reducible.

2) If $L$ is separable and for each irreducible factor $g_{i}$ of $s(L)$ it has a right factor $G_{i}$ with $s\left(G_{i}\right)=g_{i}$ then $\langle L\rangle=\cap_{i}\left\langle G_{i}\right\rangle$ is completely reducible (with $G_{i}$ being irreducible).

Proposition 1.7 For an ideal $I=<P_{1}, \ldots, P_{l}>\subset \mathcal{D}$ with at least one separable element, say $P_{1}$, one can test whether $I$ is completely reducible, by means of factoring polynomials over $F$, performing rational operations and derivating in $F$.

Proof. Trying all the factors (including reducible) of the polynomial $s\left(P_{1}\right)$ the algorithm finds all the irreducible right factors $L_{1}, \ldots, L_{k}$ of $P_{1}$ with the help of Proposition 1.2. If the ideal $I$ is completely reducible then

$$
I=<L_{i_{1}}>\cap \cdots \cap<L_{i_{n}}>
$$

for appropriate $1 \leq i_{1}<\cdots<i_{n} \leq k$.

To verify (2) the algorithm repeatedly makes use of the following procedure which finds the Janet base ([16]) of the intersection of two differential ideals $I_{1}, I_{2}$ and which extends the similar well-known procedure for finding Groebner base of the intersection of polynomial ideals [3].

Introduce the field $F(u)$ of rational functions imposing the commutativity conditions: $u \partial_{i}=$ $\partial_{i} u, 1 \leq i \leq m$ (thus, $u$ plays the role of a constant in $\mathcal{D}$ ). Find the Janet base [16] of the ideal $<u I_{1},(1-u) I_{2}>$ with respect to the lexicographical ordering in which $u$ is higher than all other variables and derivative operators. Thereupon (similar to the case of polynomial ideals [3]) all the elements of the Janet base which do not contain $u$, constitute the Janet base of $I_{1} \cap I_{2}$.

Remark 1.8 1) For an ideal $I \subset \mathcal{D}$ denote by $V_{I} \subset F$ its space of solutions [10]. The relation

$$
V_{I_{1} \cap I_{2}}=V_{I_{1}}+V_{I_{2}}
$$

(see Proposition 3.1 and Theorem 4.1 [18], cf. also Proposition 2 [2]) allows one to reduce solving a completely reducible LPDO to solving its factors.

2) If $<L>=<L_{1}>\cap<L_{2}>$ then $\operatorname{ord}(L) \leq \operatorname{ord}\left(L_{1}\right)+\operatorname{ord}\left(L_{2}\right)$.

This follows from Theorem 4.1 [18], taking into account that ord $(L)$ coincides with the leading coefficient of the Hilbert-Kolchin polynomial of the ideal $\langle L\rangle$. Moreover, ord $(L)=\operatorname{ord}\left(L_{1}\right)+$ $\operatorname{ord}\left(L_{2}\right)$ if and only if the typical differential dimension ([10]) of the gcd ideal $<L_{1}, L_{2}>\subset$ $F\left[\partial_{1}, \ldots, \partial_{m}\right]$ is less than $m-1$.

The next proposition treats the case of commuting LPDO.

Proposition 1.9 Let $L_{1} L_{2}=L_{2} L_{1}$ and $<L>=<L_{1}>\cap<L_{2}>$.

1) If $\operatorname{ord}(L)=\operatorname{ord}\left(L_{1}\right)+\operatorname{ord}\left(L_{2}\right)$ then $L=L_{1} L_{2}$.

2) If the polynomials $s\left(L_{1}\right), s\left(L_{2}\right)$ are relatively prime then $L=L_{1} L_{2}$.

Proof. To show 1) we note that $L_{1} L_{2} \in<L>$. Besides, 1) entails 2).

Below we illustrate the methods described above for the second and the third-order LPDO. 


\section{The Structure of Ideal Intersections}

In this section the possible ideal intersections which may occur if the LPDO's under consideration are from $\mathcal{D}=F\left[\partial_{x}, \partial_{y}\right]$ and their orders are not higher than three are discussed. This discussion is independent of the base field $F$ which may be $\mathbf{Q}(x, y)$, some finite extension of it or a universal field $F$, all of characteristic zero.

Theorem 2.1 Let the principal ideals $I_{1}=<\partial_{x}+a_{1} \partial_{y}+b_{1}>, I_{2}=<\partial_{x}+a_{2} \partial_{y}+b_{2}>\subset \mathcal{D}$ be different. There are three alternative cases for their intersection.

1) $I_{1} \cap I_{2}=<L=\partial_{x x}+q_{1} \partial_{x y}+q_{2} \partial_{y y}+q_{3} \partial_{x}+q_{4} \partial_{y}+q_{5}>$ and $I_{1}+I_{2}=<1>$ if and only if $a_{1}=a_{2}$. In this case $L$ is non-separable. Conversely, if $L$ is a non-separable second-order LPDO having two different first-order right factors $L_{1}, L_{2}$ then $\left.\left.\langle L\rangle=<L_{1}\right\rangle \cap<L_{2}\right\rangle$.

2) $I_{1} \cap I_{2}$ is principal and generated by a LPDO L of second-order as in the previous case and $I_{1}+I_{2} \neq<1>$, then

$$
I_{1}+I_{2}=<\partial_{x}+\frac{a_{1} b_{2}-a_{2} b_{1}}{a_{1}-a_{2}}, \partial_{y}+\frac{b_{1}-b_{2}}{a_{1}-a_{2}}>.
$$

This case occurs if and only if $\left(\frac{b_{1}-b_{2}}{a_{1}-a_{2}}\right)_{x}=\left(\frac{a_{1} b_{2}-a_{2} b_{1}}{a_{1}-a_{2}}\right)_{y}$ with $a_{1} \neq a_{2}$. In this case $L$ is separable. Conversely, if $L$ is a separable second-order LPDO having two different first-order right factors $L_{1}, L_{2}$ then $\langle L\rangle=<L_{1}>\cap<L_{2}>$.

3) $I_{1} \cap I_{2}$ is not principal, then

$$
\begin{gathered}
I_{1} \cap I_{2}=<\partial_{x x y}+q_{1} \partial_{x y y}+q_{2} \partial_{y y y}+q_{3} \partial_{x x}+q_{4} \partial_{x y}+q_{5} \partial_{y y}+q_{6} \partial_{x}+q_{7} \partial_{y}+q_{8}, \\
\partial_{x x x}+p_{1} \partial_{x y y}+p_{2} \partial_{y y y}+p_{3} \partial_{x x}+p_{4} \partial_{x y}+p_{5} \partial_{y y}+p_{6} \partial_{x}+p_{7} \partial_{y}+p_{8}>
\end{gathered}
$$

and $I_{1}+I_{2}=<1>$ if the preceding two cases do not apply.

Proof. In accordance with [3], Theorem 11 on page 186, an auxiliary parameter $u$ is introduced and the operators $u\left(\partial_{x}+a_{1} \partial_{y}+b_{1}\right)$ and $(1-u)\left(\partial_{x}+a_{2} \partial_{y}+b_{2}\right)$ are considered (see the previous section). In order to compute generators for the intersection ideal, a Janet base [16] with $u$ as the highest variable has to be computed. To this end, computationally it is more convenient to find the Janet base with respect to the differential indeterminate $z$ and a new indeterminate $w=u z$ with $w>z$ in a lexicographic term ordering. This yields the differential polynomials $w_{x}+a_{1} w_{y}+b_{1} w$ and $w_{x}+a_{2} w_{y}+b_{2} w-z_{x}-a_{2} z_{y}-b_{2} z$. If $a_{1} \neq a_{2}$ autoreduction leads to

$$
\begin{gathered}
w_{y}+\frac{b_{1}-b_{2}}{a_{1}-a_{2}} w+\frac{1}{a_{1}-a_{2}}\left(z_{x}+a_{2} z_{y}+b_{2} z\right), \\
w_{x}+\frac{a_{1} b_{2}-a_{2} b_{1}}{a_{1}-a_{2}} w-\frac{a_{1}}{a_{1}-a_{2}}\left(z_{x}+a_{2} z_{y}+b_{2} z\right) .
\end{gathered}
$$

The integrability condition between these two elements has the form

$$
\frac{b_{1}-b_{2}}{a_{1}-a_{2}} w_{x}-\frac{a_{1} b_{2}-a_{2} b_{1}}{a_{1}-a_{2}} w_{y}+\left(\left(\frac{b_{1}-b_{2}}{a_{1}-a_{2}}\right)_{x}-\left(\frac{a_{1} b_{2}-a_{2} b_{1}}{a_{1}-a_{2}}\right)_{y}\right) w+\text { terms in } z .
$$

The first derivatives $w_{x}$ and $w_{y}$ may be eliminated by a further reduction without changing the coefficient of $w$. If it vanishes, the remaining expression containing only terms in $z$ is the lowest 
element of a Janet base. The vanishing of the coefficient of $w$ yields the condition of case 2), the corresponding second-order operator generates the intersection ideal. If the coefficient of $w$ does not vanish, this expression must be applied to eliminate $w$ in (3). It yields the two third-order operators in case 3) with leading terms $\partial_{x x y}$ and $\partial_{x x x}$. Finally, if $a_{1}=a_{2}$ autoreduction leads in a single step to

$$
w_{x}+a_{1} w_{y}+b_{1} w, \quad w+\frac{1}{b_{1}-b_{2}}\left(z_{x}+a_{1} z_{y}+b_{2} z\right) .
$$

Substituting $w$ into the first expression yields the second-order operator in case 1).

To establish the last claim in case 2) apply directly Theorem 1.5.

To prove the last claim in case 1) let $L_{1}=\partial_{x}+a \partial_{y}+b_{1} \neq L_{2}=\partial_{x}+a \partial_{y}+b_{2}$ and $L=L_{3} L_{1}=L_{4} L_{2}$. Clearly, $L \in<L_{1}>\cap<L_{2}>$. Now take any $P \in<L_{1}>\cap<L_{2}>$ and divide it from the left with the remainder by $L$ with respect to $\partial_{x}$ (cf. the previous Section), then $P=Q L+R$ where $R=A \partial_{x}+B$ for certain $A, B \in F\left[\partial_{y}\right]$. Since $R=L_{5} L_{1}=L_{6} L_{2}$ we get $L_{5}=A=L_{6}$. The achieved contradiction completes the proof of the last claim in case 1 ).

Remark 2.2 a) In cases 1) and 2) of the Theorem if $L=L_{1} L_{2}$ then one can verify that $L_{1} L_{2}=$ $L_{2} L_{1}$ (the converse follows from Proposition 1.9).

b) Cases 1) and 2) of the Theorem show that the second-order LPDO L posesses the unique Loewy decomposition (which is known for the linear ordinary differential operators [12]), i.e. $L=$ $L_{1} \cdots L_{k}$ (of course, $k \leq 2$ ) where each $L_{i}$ is the lcm of its irreducible right factors. Namely, in both cases 1 ) and 2) we have $k=1$, the same holds if $L$ is irreducible. Finally, if $L$ has a unique factorization in two first-order factors $L=L_{1} L_{2}$ then $k=2$.

The above Theorem 2.1 shows that in general the $l \mathrm{~cm}$ of two LPDO's does not exist. In fact, its existence even for first-order operators is highly exceptional as it is obvious from the constraints for cases 1) and 2). It is interesting to note that in accordance with a theorem due to Stafford [19] in those cases where the $l \mathrm{~cm}$ does not exist, the intersection ideals are generated by exactly two operators, see also the discussion in [7].

The intersection of ideals generated by a LPDO of order higher than one becomes increasingly complicated and the number of alternatives increases rapidly. For an ideal $I \subset \mathcal{D}$ we denote by $L T_{I} \subset \mathcal{D}$ the leading terms ideal generated by the highest derivatives of all LPDO's from $I$ with respect to gradlex ordering in which $x>y$ (cf. [3]). The leading terms ideal $L T_{I_{1} \cap I_{2}}$ of the intersection of two ideals with $L T_{I_{1}}=<\partial_{x}>, L T_{I_{2}}=<\partial_{x x}>$ may be of different forms: for example, $<\partial_{x x x y}, \partial_{x x x x}>$, $<\partial_{x x x y y}, \partial_{x x x x}>,<\partial_{x x x}>$ etc. In the subsequent theorem the case of a principal ideal intersection will be described in some detail because it is relevant for the applications in Section 4.

Theorem 2.3 Let the principal ideals

$$
I_{1}=<\partial_{x}+a_{1} \partial_{y}+b_{1}>, I_{2}=<\partial_{x x}+a_{2} \partial_{x y}+b_{2} \partial_{y y}+c_{2} \partial_{x}+d_{2} \partial_{y}+e_{2}>\subset \mathcal{D}
$$

be given and define

$$
\begin{gathered}
p \equiv a_{1, x}-a_{1, y} a_{1}-2 a_{1} b_{1}+a_{2}\left(a_{1, y}+b_{1}\right)+a_{1} c_{2}-d_{2}, \\
q \equiv b_{1, x}-b_{1, y} a_{1}-b_{1}^{2}+a_{2} b_{1, y}+b_{1} c_{2}-e_{2}, \quad r \equiv a_{1} a_{2}-a_{1}^{2}-b_{2} .
\end{gathered}
$$

Then $L T_{I_{1} \cap I_{2}}=<\partial_{x x x}>$ if at least one of the following set of conditions (4) or (5) is satisfied.

$$
\begin{gathered}
a_{1, y y}-a_{1, y} \frac{p}{r}+2 b_{1, y}=\left(\frac{p}{r}\right)_{x}+a_{1}\left(\frac{p}{r}\right)_{y}, b_{1, y y}+b_{1, y} \frac{p}{r}-2 a_{1, y} \frac{q}{r}=\left(\frac{q}{r}\right)_{x}+a_{1}\left(\frac{q}{r}\right)_{y}, \quad r \neq 0 . \\
\left(\frac{q}{p}\right)_{x}-\left(\frac{q}{p}\right)_{y}=b_{1, y}, \quad r=0 .
\end{gathered}
$$


The proof is similar to that of Theorem 2.1 dealing with the intersection of first-order ideals and is therefore omitted. The conditions (4) or (5) are sufficient for the existence of a principal intersection. It is not excluded however, that there may be other cases involving more complicated coefficient constraints leading to a principal intersection as well.

If a third-order operator is factorized there may occur a problem of calculating the intersection of three ideals, each of which is generated by a first-order operator $L_{1}, L_{2}$ and $L_{3}$. Its intersection may be obtained by applying first Theorem 2.1 to the three pairs $\left\langle L_{i}\right\rangle \cap\left\langle L_{j}\right\rangle$ with $i=1, j=2$; $i=1, j=3$ and $i=2, j=3$. If a principal ideal is generated by some choice of $i$ and $j$, Theorem 2.3 may be applied to determine its intersection with the remaining ideal $\left\langle L_{k}\right\rangle, k \neq i, j$.

\section{Factorization of Second-Order Differential Operators}

From now on the coefficients of a given second-order operator are assumed to be from the base field $\mathbf{Q}(x, y)$. This is necessary if the goal is to obtain constructive answers allowing to factorize large classes of operators.

Theorem 3.1 (Miller 1932) Let a second-order LPDO

$$
L=\partial_{x x}+Q_{1} \partial_{x y}+Q_{2} \partial_{y y}+Q_{3} \partial_{x}+Q_{4} \partial_{y}+Q_{5}
$$

be given with $Q_{k} \in F$ for all $k$. A first order right factor $\partial_{x}+S_{1} \partial_{y}+S_{2}$ with $S_{1}, S_{2} \in F$ may exist only if there is a solution of $S_{1}^{2}-Q_{1} S_{1}+Q_{2}=0$ for $S_{1}$. The following two cases have to be distinguished.

1) $Q_{2} \neq \frac{1}{4} Q_{1}^{2}$, i.e. $L$ is separable. The two values for $S_{1}$ are $S_{1}=\frac{1}{2} Q_{1} \pm \sqrt{\frac{1}{4} Q_{1}^{2}-Q_{2}}$, then $S_{2}$ is uniquely determined from

$$
\left(S_{1}-\frac{1}{2} Q_{1}\right) S_{2}=\frac{1}{2} S_{1, x}-\frac{1}{2}\left(S_{1}-Q_{1}\right) S_{1, y}+\frac{1}{2} Q_{3} S_{1}-\frac{1}{2} Q_{4} .
$$

In addition the following condition must be satisfied.

$$
S_{2, x}-\left(S_{1}-Q_{1}\right) S_{2, y}-S_{2}^{2}+Q_{3} S_{2}-Q_{5}=0 .
$$

2) $Q_{2}=\frac{1}{4} Q_{1}^{2}$, i.e. $L$ is non-separable. There is a single value $S_{1}=\frac{1}{2} Q_{1}$, in this case $S_{2}$ has to be obtained from

$$
S_{2, x}+\frac{1}{2} Q_{1} S_{2, y}-S_{2}^{2}+Q_{3} S_{2}-Q_{5}=0 .
$$

In addition there must hold

$$
Q_{1, x}+\frac{1}{2} Q_{1} Q_{1, y}+Q_{1} Q_{3}-2 Q_{4}=0 .
$$

If there are two first-order right factors, their lcm exists and generates the same ideal as the operator (6).

Proof. Dividing the given operator (6) by $\partial_{x}+S_{1} \partial_{y}+S_{2}$, the condition that this division be exact leads to the following set of equations between the coefficients.

$$
\begin{gathered}
S_{1}^{2}-Q_{1} S_{1}+Q_{2}=0, \\
S_{1, x}+\left(Q_{1}-S_{1}\right) S_{1, y}+Q_{3} S_{1}+\left(Q_{1}-2 S_{1}\right) S_{2}=Q_{4}, \\
S_{2, x}+\left(Q_{1}-S_{1}\right) S_{2, y}-S_{2}^{2}+Q_{3} S_{2}=Q_{5} .
\end{gathered}
$$


The first equation determines always $S_{1}$. If $Q_{1} \neq 2 S_{1}$, the second equation determines $S_{2}$, and the third equation is a constraint (8) for the coefficients of (6). This is case 1). If $Q_{1}=2 S_{1}$ there holds $Q_{2}=\frac{1}{4} Q_{1}^{2}$ and the first equation has the double root $S_{1}=\frac{1}{2} Q_{1}$. The coefficient of $S_{2}$ in the second equation vanishes, upon substitution of $S_{1}$ it becomes constraint (10). $S_{2}$ has to be determined from the last equation which is (9) being a partial Riccati equation for $S_{2}$.

If there are two factors, in case 1) a straightforward but tedious calculation shows that they always obey the constraint given in case 2) of Theorem 2.1. In case 2), if two factors exist, they have identical coefficients of $\partial_{y}$ and therefore by case 1) of the same theorem the $l \mathrm{~cm}$ exists.

Remark 3.2 In the separable case 1), reducibility may always be decided, and the possible factors may be obtained by algebraic operations. Its coefficients are either from $\mathbf{Q}(x, y)$ or from a quadratic extension of $\mathbf{Q}(x, y)$. In case 2), absolute reducibility may always be decided by testing condition (10). If the answer is positive, the coefficient $S_{2}$ may be obtained by solving the partial Riccati equation (7). The latter is discussed in Lemma 5.3 in the Appendix.

Example 1. Let the operator $\partial_{x x}-\frac{2 y}{x} \partial_{x y}+\frac{y^{2}}{x^{2}}\left(1-x^{4} y^{2}\right) \partial_{y y}+\frac{2 y}{x^{2}} \partial_{y}$ be given. Because $\frac{1}{4} Q_{1}^{2}-Q_{2}=$ $x^{2} y^{4} \neq 0$, case 1 ) of Theorem 3.1 applies. It yields $S_{1}=-\frac{y}{x} \pm x y^{2}$ and $S_{2}=\mp x y$. Both choices satisfy condition (8). Consequently there exist the two first-order right factors $\partial_{x}-\left(\frac{y}{x} \pm x y^{2}\right) \partial_{y} \pm x y$.

Example 2. Consider the operator

$$
\begin{aligned}
\partial_{x x}+\frac{1}{x}\left(x^{2} y^{2}+x-y\right) \partial_{x y}+\frac{y}{x}\left(x^{2} y-1\right) \partial_{y y} & \\
& -x(y-1) \partial_{x}+\frac{1}{x^{2}}\left(x^{4} y^{2}+x^{3} y+x^{2} y^{2}-x^{2} y-x+y\right) \partial_{y}-x^{2} y-x-y .
\end{aligned}
$$

Because $\frac{1}{4} Q_{1}^{2}-Q_{2}=\frac{1}{4 x^{2}}\left(x^{2} y^{2}-x-y\right)^{2} \neq 0$, case 1) of Theorem 3.1 applies. The first alternative $S_{1}=\frac{y}{x}\left(x^{2} y-1\right)$ and $S_{2}=-x y$ satisfies condition (8). The other alternative is $S_{1}=1$ and a fairly complicated expression for $S_{2}$ which does not satisfy (8) and therefore have to be discarded. Consequently there is a single first-order right factor which leads to the factorization

$$
\left(\partial_{x}+\partial_{y}+x\right)\left(\partial_{x}-\left(\frac{y}{x}-x y^{2}\right) \partial_{y}-x y\right)
$$

of the given operator. The right factor coincides with one of the factors in the preceding example.

Example 3. (Miller 1932) Let the operator

$$
\partial_{x x}+\frac{2 y}{x} \partial_{x y}+\frac{y^{2}}{x^{2}} \partial_{y y}+\frac{1}{x} \partial_{x}+\frac{y}{x^{2}} \partial_{y}-\frac{1}{x^{2}}
$$

be given. Because $\frac{1}{4} Q_{1}^{2}-Q_{2}=0$, case 2) of Theorem 3.1 applies. It yields $S_{1}=\frac{y}{x}$ and leads to the equation $S_{2, x}+\frac{y}{x} S_{2, y}-S_{2}^{2}+\frac{1}{x} S_{2}+\frac{1}{x^{2}}=0$ for $S_{2}$. According to Lemma 5.3, its general solution is $S_{2}=\frac{1}{x} \frac{1+x^{2} \Phi(\phi)}{1-x^{2} \Phi(\phi)}$ where $\phi=\frac{y}{x}$ and $\Phi$ is an undetermined function of its argument. Consequently the given second-order operator has an infinite number of first-order right factors of the form $\partial_{x}+\frac{y}{x} \partial_{y}+\frac{1}{x} \frac{1+x^{2} \Phi(\phi)}{1-x^{2} \Phi(\phi)}$ which are parametrized by $\Phi$.

If the term $\partial_{x x}$ does not occur in LPDO (6) but $\partial_{y y}$ occurs, Theorem 3.1 may be applied after $x$ and $y$ are exchanged. The special case where the only second-order term is $\partial_{x y}$ may be traced back to the previous one by a change of variables, or it may be treated directly as in the proof of the above theorem. In any case, the answer simplifies considerably as it is shown next. 
Corollary 3.3 Let a second-order LPDO $\partial_{x y}+Q_{3} \partial_{x}+Q_{4} \partial_{y}+Q_{5}$ be given with $Q_{k} \in \mathbf{Q}(x, y)$ for all $k$. The following two cases have to be distinguished.

1) There exists a first-order right factor $\partial_{x}+Q_{4}$ if and only if $Q_{5}-Q_{3} Q_{4}=Q_{4, y}$.

2) There exists a first-order right factor $\partial_{y}+Q_{3}$ if and only if $Q_{5}-Q_{3} Q_{4}=Q_{3, x}$.

If the conditions for both cases 1) and 2) are satisfied, the given LPDO is the lcm of the two first-order factors.

Example 4. Let a LPDO $\partial_{x y}+\frac{1}{x+y}\left(\alpha \partial_{x}+\beta \partial_{y}\right)$ be given. The preceding corollary leads immediately to the following result. If $\alpha=1, \beta \neq 1$ it factors into $\left(\partial_{y}+\frac{1}{x+y}\right)\left(\partial_{x}+\frac{\beta}{x+y}\right)$. If $\alpha \neq 1$ and $\beta=1$ it factors into $\left(\partial_{x}+\frac{1}{x+y}\right)\left(\partial_{y}+\frac{\alpha}{x+y}\right)$. If both $\alpha=\beta=1$ it is equal to $\operatorname{lcm}\left(\partial_{x}+\frac{1}{x+y}, \partial_{y}+\frac{1}{x+y}\right)$.

If a second-order LPDO is reducible, solving the corresponding differential equation is reduced to solving one or more first order equations (cf. the Remark 1.8). In the Appendix a few results on solving such equations are listed. They are applied next for solving reducible second-order equations.

Theorem 3.4 The solutions of a second-order linear pde may be described as follows.

1) If the corresponding second-order LPDO is the lcm of two first-order LPDO, i.e. if

$$
L \equiv \partial_{x x}+q_{1} \partial_{x y}+q_{2} \partial_{y y}+q_{3} \partial_{x}+q_{4} \partial_{y}+q_{5}=\operatorname{lcm}\left(\partial_{x}+a_{1} \partial_{y}+a_{2}, \partial_{x}+b_{1} \partial_{y}+b_{2}\right),
$$

the general solution of $L z=0$ has the form $z(x, y)=z_{0}(x, y)+z_{1}(x, y)$ (see the Remark 1.8) where

$$
z_{0}(x, y)=\left.\Phi(\phi) e^{-\int a_{2}(x, \bar{\phi}) d x}\right|_{\bar{y}=\phi}, \quad z_{1}(x, y)=\left.\Psi(\psi) e^{-\int b_{2}(x, \bar{\psi}) d x}\right|_{\bar{y}=\psi},
$$

$\phi(x, y)$ is the integral of $\frac{d y}{d x}=a_{1}, \psi(x, y)$ is the integral of $\frac{d y}{d x}=b_{1}, \bar{\phi}$ is the inverse of $\bar{y}=$ $\phi(x, y)$, i. e. $y=\bar{\phi}(x, \bar{y})$, and $\bar{\psi}$ is the inverse of $\bar{y}=\psi(x, y)$, i. e. $y=\bar{\psi}(x, \bar{y})$.

If the gcd ideal of the two first-order factors is nontrivial with a solution $w(x, y)$, by Corollary 5.2, the general solution may be written in the simplified form $[\Phi(\phi)+\Psi(\psi)] w(x, y)$. If the gcd ideal is finite-dimensional then in order to yield its solution $w(x, y)$ one can make use of [11].

2) If the corresponding second-order LPDO is not the lcm of two first-order LPDO but has only one factorization

$$
L \equiv \partial_{x x}+q_{1} \partial_{x y}+q_{2} \partial_{y y}+q_{3} \partial_{x}+q_{4} \partial_{y}+q_{5}=\left(\partial_{x}+b_{1} \partial_{y}+b_{2}\right)\left(\partial_{x}+a_{1} \partial_{y}+a_{2}\right),
$$

the general solution of $L z=0$ has the form

$$
z(x, y)=z_{0}(x, y)+\left.e^{-\int a_{2}(x, \bar{\phi}) d x} \int z_{1}(x, \bar{\phi}) e^{\int a_{2}(x, \bar{\phi}) d x} d x\right|_{\bar{y}=\phi}
$$

with $z_{0}$ and $z_{1}$ as in the previous case.

Example 1 (completed). By case 2) of Theorem 2.1, the $g c d$ ideal is $\left\langle\partial_{x}-\frac{1}{x}, \partial_{y}-\frac{1}{y}>\neq 1\right.$. With the notation of case 1) of Theorem 3.4, the first order ode's $y^{\prime} \pm x y^{2}+\frac{1}{x} y=0$ for the integral of the homogeneous part are obtained. These Riccati equations have the general solutions $y=\frac{1}{C x \pm x^{2}}$ 
where $C$ is a constant. They yield the first integrals $\phi=\frac{1}{x y}+x$ and $\psi=\frac{1}{x y}-x$ respectively. Applying Corollary 5.2 the general solution may be written as $\left[\Phi\left(\frac{1}{x y}+x\right)+\Psi\left(\frac{1}{x y}-x\right)\right] x y$.

Example 2 (completed). The solution has to be constructed by case 2) of Theorem 3.4. The right factor yields $z_{0}(x, y)=x y \Phi\left(\frac{1}{x y}+x\right)$, the left factor $z_{1}(x, y)=\Psi(x-y) \exp \left(-\frac{1}{2} x^{2}\right)$ with $\Phi$ and $\Psi$ undetermined functions. Substituting these expressions into (13) finally yields

$$
z(x, y)=x y \Phi\left(\frac{1}{x y}+x\right)-\left.\frac{1}{x y} \int(x-\bar{y}) \Psi(x-\bar{\phi}) \exp \left(-\frac{1}{2} x^{2}\right) d x\right|_{\bar{y}=\phi}
$$

where $\phi=\frac{1}{x y}+x$ and $\bar{\phi}=\frac{1}{x(\bar{y}-x)}$.

Example 3 (completed). There are several ways to proceed in this case. Two different right factors may be created by two choices of $\Phi$, e. g. $\Phi=1$ and $\Phi=\frac{y}{x}$. Then by applying case 1) of Theorem 3.4, the solution is obtained in the form

$$
z(x, y)=\left(x-\frac{1}{x}\right) \Phi\left(\frac{y}{x}\right)+\frac{y}{x^{2}}(x y-1) \Psi\left(\frac{y}{x}\right) .
$$

Alternatively Lemma 5.1 may be applied to the general first-order factor which results in

$$
z(x, y)=\frac{1}{x} \Phi\left(\frac{y}{x}\right)\left(x^{2} \Phi\left(\frac{y}{x}\right)-1\right) \Psi\left(\frac{y}{x}\right)=x \bar{\Phi}\left(\frac{y}{x}\right)-\frac{1}{x} \bar{\Psi}
$$

where $\bar{\Phi}=\Phi^{2} \Psi$ and $\bar{\Psi}=\Phi \Psi$.

\section{Factorization of Third-Order Differential Operators}

In this section we study third-order LPDO's from the ring $\mathcal{D}=\mathbf{Q}(x, y)\left[\partial_{x}, \partial_{y}\right]$.

Theorem 4.1 Let a third-order LPDO be given with coefficients $Q_{k} \in F$ for all $k$.

$$
\partial_{x x x}+Q_{1} \partial_{x x y}+Q_{2} \partial_{x y y}+Q_{3} \partial_{y y y}+Q_{4} \partial_{x x}+Q_{5} \partial_{x y}+Q_{6} \partial_{y y}+Q_{7} \partial_{x}+Q_{8} \partial_{y}+Q_{9} .
$$

A first-order right factor $\partial_{x}+S_{1} \partial_{y}+S_{2}$ with $S_{1}, S_{2} \in F$ may exist only if there is a solution of $S_{1}^{3}-Q_{1} S_{1}^{2}+Q_{2} S_{1}-Q_{3}=0$ in $F$ for $S_{1}$. In addition, the following two cases have to be distinguished.

1.1) If $S_{1}^{2}-\frac{2}{3} Q_{1} S_{1}+\frac{1}{3} Q_{2} \neq 0$ (cf. Remark 1.3), then $S_{2}$ is uniquely determined from

$$
\begin{aligned}
& \left(S_{1}^{2}-\frac{2}{3} Q_{1} S_{1}+\frac{1}{3} Q_{2}\right) S_{2} \\
& \quad=\left(S_{1}-\frac{1}{3} Q_{1}\right) S_{1, x}-\left(S_{1}^{2}-Q_{1} S_{1}+\frac{2}{3} Q_{2}\right) S_{1, y}+\frac{1}{3} Q_{4} S_{1}^{2}-\frac{1}{3} Q_{5} S_{1}+\frac{1}{3} Q_{6} .
\end{aligned}
$$

1.2) If $S_{1}^{2}-\frac{2}{3} Q_{1} S_{1}+\frac{1}{3} Q_{2}=0$ (i.e. (14) being non-separable, cf. Section 1), then $S_{2}$ has to be obtained as a solution of

$$
\begin{aligned}
\left(S_{1}-\right. & \left.\frac{1}{3} Q_{1}\right) S_{2, x}-\left(S_{1}^{2}-Q_{1} S_{1}+\frac{2}{3} Q_{2}\right) S_{2, y}-\left(S_{1}-\frac{1}{3} Q_{1}\right) S_{2}^{2} \\
& +\left(S_{1, x}-S_{1} S_{1, y}+\frac{2}{3} Q_{1} S_{1, y}+\frac{2}{3} Q_{4} S_{1}-\frac{1}{3} Q_{5}\right) S_{2} \\
& -\frac{1}{3} S_{1, x x}+\frac{1}{3}\left(S_{1}-Q_{1}\right) S_{1, x y}-\frac{1}{3}\left(S_{1}^{2}-Q_{1} S_{1}+Q_{2}\right) S_{1, y y}+\frac{2}{3} S_{1, x} S_{1, y} \\
& -\frac{1}{3} Q_{4} S_{1, x}-\frac{1}{3}\left(S_{1}-Q_{1}\right) S_{1, y}^{2}+\frac{1}{3}\left(Q_{4} S_{1}-Q_{5}\right) S_{1, y}-\frac{1}{3} Q_{7} S_{1}+\frac{1}{3} Q_{8}=0 .
\end{aligned}
$$


Two additional constraints of the form

$$
F_{k}\left(Q_{1}, \ldots, Q_{9}, Q_{1, x}, \ldots, Q_{9, y y}\right)=0 \text { for } k=1,2
$$

and $F_{k}$ a polynomial in its arguments have to be satisfied. Due to their huge sizes we omit them.

A second-order right factor $\partial_{x x}+S_{1} \partial_{x y}+S_{2} \partial_{y y}+S_{3} \partial_{x}+S_{4} \partial_{y}+S_{5}$ of (14) may exist only if there is a solution of $S_{1}^{3}-2 Q_{1} S_{1}^{2}+\left(Q_{2}+Q_{1}^{2}\right) S_{1}+Q_{3}-Q_{1} Q_{2}=0$ for $S_{1}$. Then $S_{2}=S_{1}^{2}-Q_{1} S_{1}+Q_{2}$ and the following two cases have to be distinguished.

2.1) If $S_{1}^{2}-\frac{4}{3} Q_{1} S_{1}+\frac{1}{3}\left(Q_{2}+Q_{1}^{2}\right) \neq 0$, then $S_{3}$ is uniquely determined from

$$
\begin{aligned}
\left(S_{1}^{2}-\right. & \left.\frac{4}{3} Q_{1} S_{1}+\frac{1}{3}\left(Q_{2}+Q_{1}^{2}\right)\right) S_{3}+\left(S_{1}-\frac{2}{3} Q_{1}\right) S_{1, x}-\left(S_{1}^{2}-\frac{5}{3} Q_{1} S_{1}-\frac{2}{3} Q_{1}^{2}\right) S_{1, y} \\
& +\frac{1}{3}\left(2 Q_{4}+Q_{1, y}\right) S_{1}^{2}-\frac{1}{3}\left(Q_{5}+2 Q_{1} Q_{4}+Q_{2, y}+Q_{1, x}+Q_{1} Q_{1, y}\right) S_{1} \\
& +\frac{1}{3}\left(Q_{2, x}+Q_{2, y} Q_{1}+Q_{4} Q_{2}+Q_{5} Q_{1}-Q_{6}\right)=0
\end{aligned}
$$

2.2) If $S_{1}^{2}-\frac{4}{3} Q_{1} S_{1}+\frac{1}{3}\left(Q_{2}+Q_{1}^{2}\right)=0$, then $S_{3}$ is obtained from

$$
\begin{aligned}
\left(S_{1}-\right. & \left.\frac{2}{3} Q_{1}\right) S_{3, x}-\left(S_{1}^{2}-\frac{5}{3} Q_{1} S_{1}+\frac{2}{3} Q_{1}^{2}\right) S_{3, y}-\left(S_{1}-\frac{2}{3} Q_{1}\right) S_{3}^{2} \\
& +\left(S_{1, x}-S_{1} S_{1, y}+Q_{1} S_{1, y}+\frac{4}{3} Q_{4} S_{1}+\frac{1}{3} Q_{1, y} S_{1}-\frac{1}{3} Q_{5}-\frac{2}{3} Q_{4} Q_{1}-\frac{1}{3} Q_{1, x}-\frac{1}{3} Q_{1} Q_{1, y}\right) S_{3} \\
& \quad-\frac{1}{3} S_{1, x x}+\frac{2}{3}\left(S_{1}-Q_{1}\right) S_{1, x y}-\frac{1}{3}\left(S_{1}^{2}-2 Q_{1} S_{1}+Q_{1}^{2}\right) S_{1, y y}+\frac{1}{3} S_{1, x} S_{1, y}+\frac{2}{3} Q_{4}\left(S_{1, y} S_{1}-S_{1, x}\right) \\
& -\frac{2}{3} Q_{4} S_{1, x}-\frac{1}{3}\left(S_{1}-Q_{1}\right) S_{1, y}^{2}+\frac{1}{3}\left(Q_{1, y} S_{1}-2 Q_{4} Q_{1}-Q_{1, x}-Q_{1, y} Q_{1}\right) S_{1, y}+\frac{1}{3} Q_{4, y} S_{1}^{2} \\
& -\frac{1}{3}\left(Q_{7}+Q_{5, y}+Q_{4, x}+Q_{4, y} Q_{1}+Q_{4}^{2}\right) S_{1}+\frac{1}{3}\left(Q_{8}+Q_{7} Q_{1}+Q_{5, x}+Q_{5, y} Q_{1}+Q_{5} Q_{4}\right)=0 .
\end{aligned}
$$

As for the first-order factor, two additional constraints of the form (17) have to be satisfied.

The remaining coefficients are

$$
\begin{gathered}
S_{4}=2 S_{3} S_{1}-Q_{1} S_{3}-S_{1, x}+\left(S_{1}-Q_{1}\right) S_{1, y}-Q_{4} S_{1}+Q_{5}, \\
S_{5}=S_{3}^{2}-S_{3, x}+\left(S_{1}-Q_{1}\right) S_{3, y}-Q_{4} S_{3}+Q_{7} .
\end{gathered}
$$

The proof of this result is similar to the case of the second-order factorization in Theorem 3.1 and is therefore omitted.

Remark 4.2 In the separable cases 1.1) and 2.1), reducibility may always be decided, and the possible factors may be obtained by algebraic operations and derivations. Its coefficients are either from $\mathbf{Q}(x, y)$ or from a quadratic or cubic extension of it. In cases 1.2) and 2.2), absolute reducibility may always be decided by testing conditions (17). If absolute irreducibility is precluded, the coefficient $S_{2}$ or $S_{3}$ may be obtained by solving the partial Riccati equation (16) or (19) respectively.

Example 5 (Blumberg 1912) In his dissertation [1] Blumberg gave the following example of a reducible third-order LPDO with two different factorizations involving first-order right factors.

$$
\begin{aligned}
L= & \partial_{x x x}+x \partial_{x x y}+2 \partial_{x x}+2(x+1) \partial_{x y}+\partial_{x}+(x+2) \partial_{y} \\
& =\left(\partial_{x x}+x \partial_{x y}+\partial_{x}+(x+2) \partial_{y}\right)\left(\partial_{x}+1\right)=\left(\partial_{x}+1\right)\left(\partial_{x}+1\right)\left(\partial_{x}+x \partial_{y}\right) .
\end{aligned}
$$


The right factor $\partial_{x}+1$ is obtained from case 1.2) of the above theorem. The solution $S_{1}=0$ leads to the equation

$$
S_{2, x}-S_{2}^{2}+\left(2+\frac{2}{x}\right) S_{2}-1-\frac{2}{x}=0
$$

with the general solution $S_{2}=1+\frac{1}{x}-\frac{1}{C+x}$ where $C$ denotes an arbitrary function depending only on $y$. The constraint $F_{1}=0$ is satisfied for any $C$ whereas $F_{2}=0$ leads to $C=0$, i. e. $S_{2}=1$. Furthermore Blumberg showed that the second-order left factor in this factorization is absolutely irreducible. The other right factor $\partial_{x}+x \partial_{y}$ is obtained from case 1 ) for $S_{1}=x$. It turns out that the quotient by this factor which is $\partial_{x x}+2 \partial_{x}+1$ is completely reducible with the consequence that the second factorization in (20) should be replaced by

$$
l c m\left(\partial_{x}+1-\frac{1}{C_{1}+x}, \partial_{x}+1-\frac{1}{C_{2}+x}\right)\left(\partial_{x}+x \partial_{y}\right)
$$

where $C_{1} \neq C_{2}$ are constants w.r.t. $x$, i. e. $\left(C_{k}\right)_{x}=0, k=1,2$. The limit $C_{k} \rightarrow \infty$ leads to the factor $\partial_{x}+1$ as given in (20). The intersection ideal of the two first-order right factors

$$
\begin{aligned}
<\partial_{x}+1>\cap & <\partial_{x}+x \partial_{y}>= \\
< & \partial_{x x y}+x \partial_{x y y}-\frac{1}{x} \partial_{x y}+x \partial_{y y}-\frac{1}{x} \partial_{x}-\frac{x+1}{x} \partial_{y}, \\
& \partial_{x x x}-x^{2} \partial_{x y y}+3 \partial_{x x}+(2 x+3) \partial_{x y}-x^{2} \partial_{y y}+2 \partial_{x}+(2 x+3) \partial_{y}>
\end{aligned}
$$

is not principal (see Theorem 2.1 case 3)). This demonstrates that a direct analogue of the Loewy decomposition (cf. [12] and the Remark 2.2) fails for third-order LPDO's. Indeed, $L$ from (20) is not lcm of its only two irreducible right factors $\partial_{x}+1$ and $\partial_{x}+x \partial_{y}$ (one can check making use of Theorem 4.1 that there are no other irreducible right factors neither of the first- nor of the secondorder). However, all intersections

$$
<\left(\partial_{x}+1-\frac{1}{C+x}\right)\left(\partial_{x}+x \partial_{y}\right)>\cap<\partial_{x}+1>=<L>
$$

are principal. This means that $L$ has infinitely many representations as the $l \mathrm{~cm}$ of a second-order and a first-order operator.

Similar as in the second-order case, when the leading derivative of a third-order operator is $\partial_{x x y}$ or $\partial_{x y y}$, the possible factorizations are relatively simple as is shown next for the former case. The latter may be reduced to it by exchanging its variables.

Corollary 4.3 Let a third-order LPDO $\partial_{x x y}+Q_{2} \partial_{x y y}+Q_{4} \partial_{x x}+Q_{5} \partial_{x y}+Q_{6} \partial_{y y}+Q_{7} \partial_{x}+Q_{8} \partial_{y}+Q_{9}$ be given with $Q_{k} \in \mathbf{Q}(x, y)$ for all $k$. The following four cases have to be distinguished.

1) There exists a first-order right factor $\partial_{x}+S_{1} \partial_{y}+S_{2}$ if and only if one of the following set of conditions is satisfied.

$$
\begin{gathered}
S_{1}=0, \quad S_{2}=\frac{Q_{6}}{Q_{2}}, \quad S_{2, x}+2 Q_{2} S_{2, y}-S_{2}^{2}+Q_{5} S_{2}=Q_{8}, \\
S_{2, x y}+Q_{2} S_{2, y y}+Q_{4} S_{2, x}+Q_{5} S_{2, y}-Q_{4} S_{2}^{2}+Q_{7} S_{2}=2 S_{2} S_{2, y}
\end{gathered}
$$

or

$$
\begin{gathered}
S_{1}=Q_{2}, \quad S_{2}=-\frac{Q_{6}}{Q_{2}}+\frac{Q_{2, x}}{Q_{2}}-Q_{2, y}-Q_{2} Q_{4}+Q_{5} \\
S_{2, x y}+Q_{4} S_{2, x}+\left(Q_{5}-Q_{2, y}-Q_{2} Q_{4}\right) S_{2, y}-Q_{4} S_{2}^{2}+Q_{7} S_{2}=2 S_{2, y} S_{2}+Q_{9} \\
S_{2, x}-S_{2}^{2}+\left(Q_{5}-Q_{2} Q_{4}-Q_{2, y}\right) S_{2}+Q_{2, y y} Q_{2}+\left(Q_{4, y}+Q_{4}^{2}\right) Q_{2}^{2} \\
+\left(2 Q_{2, y}-Q_{5}\right) Q_{2} Q_{4}-Q_{2} Q_{5, y}+Q_{2} Q_{7}+Q_{4} Q_{6}+Q_{6, y}-Q_{8}=0 .
\end{gathered}
$$


2) There exists a first-order right factor $\partial_{y}+S_{2}$ if and only if there holds

$$
\begin{gathered}
S_{2}=Q_{4}, \quad 2 Q_{4, x}+Q_{2} Q_{4, y}+Q_{4}\left(Q_{5}-Q_{2} Q_{4}\right)=Q_{7}, \\
Q_{4, x x}+Q_{2} Q_{4, x y}+\left(Q_{5}-2 Q_{2} Q_{4}\right) Q_{4, x}+Q_{6} Q_{4, y}=Q_{4}\left(Q_{4} Q_{6}-Q_{8}\right)+Q_{9} .
\end{gathered}
$$

3) There exists a second-order right factor $\partial_{x x}+S_{1} \partial_{x y}+S_{2} \partial_{y y}+S_{3} \partial_{x}+S_{4} \partial_{y}+S_{5}$ if and only if there holds

$$
\begin{gathered}
S_{1}=Q_{2}, \quad S_{2}=0, \quad S_{3}=Q_{5}-Q_{2} Q_{4}-Q_{2, y}, \quad S_{4}=Q_{6}, \quad S_{5}=Q_{8}-Q_{4} Q_{6}-Q_{6, y}, \\
S_{3, y}+Q_{4} S_{3}=Q_{7}, \quad S_{5, y}+Q_{4} S_{5}=Q_{9} .
\end{gathered}
$$

4) There exists a second-order right factor $\partial_{x y}+S_{2} \partial_{y y}+S_{3} \partial_{x}+S_{4} \partial_{y}+S_{5}$ if and only if one of the following set of conditions is satisfied.

$$
\begin{gathered}
S_{2}=0, \quad S_{3}=Q_{4}, \quad S_{4}=\frac{Q_{6}}{Q_{2}}, \quad S_{5}=\frac{Q_{4} Q_{6}}{Q_{2}}-Q_{2}\left(Q_{4, y}-Q_{4}^{2}\right)-Q_{4} Q_{5}-Q_{4, x}+Q_{7}, \\
S_{4, x}+Q_{2} S_{4, y}+Q_{5} S_{4}-S_{4}^{2}+Q_{2} S_{5}=Q_{4} Q_{6}+Q_{8}, \\
S_{5, x}+Q_{2} S_{5, y}+\left(Q_{5}-Q_{2} Q_{5}\right) S_{5}-S_{4} S_{5}=Q_{9}
\end{gathered}
$$

or

$$
\begin{gathered}
S_{2}=Q_{2}, \quad S_{3}=Q_{4}, \quad S_{4}=\frac{Q_{2, x}}{Q_{2}}+Q_{5}-\frac{Q_{6}}{Q_{2}}, \quad S_{5}=\frac{Q_{4}}{Q_{2}}\left(Q_{2, x}-Q_{6}-Q_{4, x}+Q_{7}\right), \\
S_{4, x}-S_{4}^{2}+Q_{5} S_{4}=Q_{8}, \quad S_{5, x}+\left(Q_{5}-S_{4}\right) S_{5}=Q_{9} .
\end{gathered}
$$

\section{Conclusion}

The factorization problem for second- and third-order differential operators in two variables has been shown to require the solution of a partial Riccati equation (23), which in turn requires to solve a general first-order ode and possibly an ordinary Riccati equation. The bottleneck for designing a factorization algorithm for a LPDO is the general first-order ode which makes the full problem intractable at present because in general there are no solution algorithms available. Some of the results described in this article may be generalized to any number of independent variables.

\section{Appendix}

The subject of this appendix are quasilinear first-order partial differential equations in a single dependent variable $z$, depending on $x$ and $y$. These results are needed in the main text, they are due to Goursat [4] and Kamke [9]. The first lemma deals with the general linear equation.

Lemma 5.1 The general solution of the linear partial differential equation

$$
z_{x}+a_{1} z_{y}+a_{2} z+a_{3}=0
$$

with $a_{k} \equiv a_{k}(x, y) \in \mathbf{Q}(x, y), k=1, \ldots, 3$ may be described as follows. Let $\phi(x, y)=$ const be the first integral obtained from the solution of the first order ode $\frac{d y}{d x}=a_{1}(x, y)$. Assume that $\bar{y}=\phi(x, y)$ and the inverse $y=\bar{\phi}(x, \bar{y})$ exist. Then the general solution of (21) is

$$
z(x, y)=\left.\left(\Phi(\phi)-\int a_{3}(x, \bar{\phi}) e^{\int a_{2}(x, \bar{\phi}) d x} d x\right) e^{-\int a_{2}(x, \bar{\phi}) d x}\right|_{\bar{y}=\phi}
$$

with $\Phi$ an undetermined function of its argument. 
Proof. It is based on the procedure described in [9], Section 4.2. A homogeneous equation corresponding to (21) for a new function $w(x, y, z)$ is $w_{x}+a_{1} w_{y}-\left(a_{2} z+a_{3}\right) w_{z}=0$. A first integral $\phi$ is obtained from $\frac{d y}{d x}=a_{1}$. If it is applied for introducing a new variable $\bar{y}$ as described in the Lemma, the equation $\bar{w}_{x}-\left.\left(a_{2} z+a_{3}\right)\right|_{y=\bar{\phi}} \bar{w}_{z}=0$ for $\bar{w}$ is obtained with the first integral

$$
\psi=z e^{\int a_{2}(x, \bar{\phi}) d x}+\int a_{3}(x, \bar{\phi}) e^{\int a_{2}(x, \bar{\phi}) d x} d x .
$$

Consequently the general solution $w=\Psi(\phi, \psi)$ is obtained with an undetermined function $\Psi$. Resubstituting $\bar{y}=\phi(x, y)$ and solving for $z$ yields $(22)$.

Corollary 5.2 Let $z_{1} \neq 0$ and $z_{2}$ be special solutions of $z_{x}+a_{1} z_{y}+a_{2} z=0$, then the quotient $z_{0}=\frac{z_{2}}{z_{1}}$ is a solution of the homogeneous equation $z_{x}+a_{1} z_{y}=0$.

Proof. For any two solutions $z_{1}, z_{2}$ of $z_{x}+a_{1} z_{y}+a_{2} z=0$, the quotient $\frac{z_{2}}{z_{1}}$ is a solution of $z_{x}+a_{1} z_{y}=0$ as is obvious from

$$
\left(\frac{z_{2}}{z_{1}}\right)_{x}+a_{1}\left(\frac{z_{2}}{z_{1}}\right)_{y}=\frac{1}{z_{1}}\left(z_{2, x}+a_{1} z_{2, y}\right)-\frac{z_{2}}{z_{1}^{2}}\left(z_{1, x}+a_{1} z_{1, y}\right)=\frac{1}{z_{1}}\left(-a_{2} z_{1}\right)-\frac{z_{2}}{z_{1}^{2}}\left(-a_{2} z_{1}\right)=0 .
$$

For $a_{2}$ and/or $a_{3}=0$, the solution (22) simplifies in an obvious way to the solution of the respective homogeneous equation.

The equation considered in the next Lemma is called partial Riccati equation for obvious reasons, i. e. it is linear in the first order derivatives and quadratic in the dependent variable.

Lemma 5.3 The general solution of the partial Riccati equation

$$
z_{x}+a_{1} z_{y}+a_{2} z^{2}+a_{3} z+a_{4}=0
$$

with $a_{k} \equiv a_{k}(x, y) \in \mathbf{Q}(x, y), k=1, \ldots, 4$, may be described as follows. Let $\phi(x, y)=$ const be the first integral obtained from the solution of the first order ode $\frac{d y}{d x}=a_{1}(x, y)$. Assume that $\bar{y}=\phi(x, y)$ and the inverse $y=\bar{\phi}(x, \bar{y})$ exists, and generate the Riccati equation

$$
\frac{d z}{d x}+a_{2}(x, \bar{y}) z^{2}+a_{3}(x, \bar{y}) z+a_{4}(x, \bar{y})=0 .
$$

Into its solution substitute $\bar{y}=\phi(x, y)$ to obtain the first integral $\psi(x, y, z)=$ const. Solving the relation $\psi=\Phi(\phi)$ for $z$, where $\Phi$ is an undetermined function of its argument, yields the general solution of (23).

Proof. By the same procedure as in the proof for Lemma 5.1, now for $\bar{w}(x, \bar{y}, z)$ the equation $\bar{w}_{x}-\left.\left(a_{2} z^{2}+a_{3} z+a_{4}\right)\right|_{y=\bar{\phi}} \bar{w}_{z}=0$ is obtained. Its first integral is determined by the general solution of (24), from which the solution of (23) is obtained as described above.

Remark 5.4 Both equations (21) and (23) require solving a first order non-linear ode. No algorithm is known for solving such an equation in general. If any solution has been found by ad hoc methods, e. g. using a symmetry, the solution (22) is obtained by integration.

Acknowledgements. The paper was done during a stay of the first author at the Institut SCAI of the Fraunhofer Gesellschaft, supported by the Forschungspreis of the Humboldt Foundation which is gratefully acknowldeged. The authors are grateful to Mike Singer, Serguei Tsarev and Eckehart Hotzel for useful discussions. 


\section{References}

[1] H. Blumberg, Über algebraische Eigenschaften von linearen homogenen Differentialausdrücken, Inaugural-Dissertation, Göttingen, 1912,

[2] P. Cassidy, Differential Algebraic Groups, Amer. J. Math., 94, 891-954 (1972).

[3] D. Cox, J. Little, D. O’Shea, Ideals, Varieties and Algorithms, Springer, 1991.

[4] E. Goursat, Lecons sur l'integration des équations aux déeriveées partielles du premier ordre, Gauthier-Villars et $\mathrm{C}^{i e}$, Paris, 1921.

[5] D. Grigoriev, Complexity of Factoring and Calculating the GCD of Linear Ordinary Differential Operators, J. Symbolic Computation, 7, 7-37 (1990).

[6] D. Grigoriev, Complexity of Solving Systems of Linear Equations over Rings of Differential Operators, Progress in Math., Birkhauser, 94, 195-202 (1991)

[7] A. Hillebrand, W. Schmale, Towards an Effective Version of a Theorem due to Stafford, J. Symbolic Computation, 32, 699-716 (2001).

[8] E. Kaltofen, Factorization of Polynomials, in Computing Supplementum 4, B. Buchberger, G. E. Collins and R. Loos Edts., page 95-113, 1982.

[9] E. Kamke, Differentialgleichungen: Lösungsmethoden und Lösungen, II. Partielle Differentialgleichungen, Akademische Verlagsgesellschaft, Leipzig, 1965.

[10] E. Kolchin, Differential Algebra and Algebraic Groups, Academic Press, 1973.

[11] Z. Li, F. Schwarz, S. Tsarev, Factoring systems of linear PDE's with finite-dimensional solution space, J. Symbolic Comput., 36, 443-471 (2003).

[12] A. Loewy, Über vollständig reduzible lineare homogene Differentialgleichungen, Mathematische Annalen, 62, 89-117 (1906).

[13] F. H. Miller, Reducible and irreducible linear differential operators, $\mathrm{PhD}$ Thesis, Columbia University, 1932.

[14] M. van der Put, M. Singer, Galois theory of linear differential equations, Grundlehren der Mathematischen Wissenschaften, 328, Springer, 2003.

[15] L. Schlesinger, Handbuch der Theorie der linearen Differentialgleichungen, Leipzig, Teubner, 1897.

[16] F. Schwarz, Janet bases for symmetry groups, Groebner bases and applications, in London Math. Society, Lecture Note Ser., 251, Cambridge University Press, 221-234 (1998).

[17] F. Schwarz, A Factorization Algorithm for Linear Ordinary Differential Equations, Proceedings of the ISSAC'89, page 17-25, ACM Press, 1989, Gaston Gonnet, ed.

[18] W. Sit, Typical Differential Dimension of the Intersection of Linear Differential Algebraic Groups, Journal of Algebra 32, 476-487 (1974).

[19] J. T. Stafford, Module Structure of Weyl Algebras, J. London Math. Soc., Ser II, 18, 429-442 (1978). 
[20] S. Tsarev, Factorization of linear partial differential operators and the Darboux method for integrating nonlinear partial differential equations, Theoret. and Math. Phys., 122, 121-133 (2000).

Dima Grigoriev

CNRS, IRMAR

Université de Rennes, Beaulieu

35042, Rennes, France

Email: dima@math.univ-rennes.fr

Website: http://name.math.univ-rennes1.fr/dimitri.grigoriev

Fritz Schwarz

FhG, Institut SCAI

53754 Sankt Augustin, Germany

Email: fritz.schwarz@scai.fraunhofer.de

Website: www.scai.fhg.de/schwarz.html 